\title{
ioFog: Prediction-based Fog Computing Architecture for Offline IoT
}

\author{
Mehbub Alam ${ }^{1}$, Nurzaman Ahmed ${ }^{2}$, Rakesh Matam ${ }^{3}$, and Ferdous Ahmed Barbhuiya ${ }^{4}$ \\ ${ }^{1,3,4}$ Department of Computer Science \& Engineering \\ Indian Institute of Information Technology Guwahati \\ ${ }^{2}$ Department of Computer Science \& Engineering \\ Indian Institute of Technology Kharagpur \\ ${ }^{1}$ mehbub@iiitg.ac.in ${ }^{2}$ nurzaman@cse.iitkgp.ac.in, ${ }^{3}$ rakesh@iiitg.ac.in, ${ }^{4}$ ferdous@iiitg.ac.in
}

\begin{abstract}
Due to the multi-hop, long-distance, and wireless backbone connectivity, provisioning critical and diverse services face challenges such as low latency and reliability. This paper proposes ioFog, an offline fog architecture for achieving reliability and low latency in a large backbone network. Our solution uses a Markov chain-based task prediction model to offer dynamic service requirements with minimal dependency on the Internet. The proposed architecture considers a central Fog Controller (FC) to (i) provide a global status overview and (ii) predict upcoming tasks of Fog Nodes for intelligent offloading decisions. The FC also has the current status of the existing fog nodes in terms of their processing and storage capabilities. Accordingly, it can schedule the possible future offline computations and task allocations. ioFog considers the requirements of individual IoT applications and enables improved fog computing decisions. As compared to the existing offline IoT solutions, ioFog improves service time significantly and service delivery ratio up to $23 \%$.

Index Terms-Offline IoT, Markov chain model, Fog computing, Internet of Things.
\end{abstract}

\section{INTRODUCTION}

With the growing size of the wireless backbone network, maintaining continuous Internet connectivity and low latency services is a challenge [1]. While provisioning immediate decision and control of critical IoT applications, the current state-of-the-art solutions are not sufficient. Existing offline IoT solutions such as [2] and [3] provide connectivity using edgebased architecture. These solutions do not consider backbone network connectivity issues; consequently, they are deficient in providing reliable and low latency services. Moreover, the current fog-based task offloading solutions require a huge amount of control overhead. Therefore, a new computing architecture is essential to control and monitor fog networks and their services.

This paper proposes ioFog, an fog computing architecture for offline IoT operations. A fog controller module (FC) in the network monitors and controls different fog and cloud activities for improved service providing and decision making. With a prediction module at the FC, the proposed network architecture provisions offline connectivity to various critical IoT services. As shown in Fig. 1, the proposed ioFog network uses backbone nodes for fog and control operations. With the help of a global overview, the FC chooses a Fog node and executes a new task.

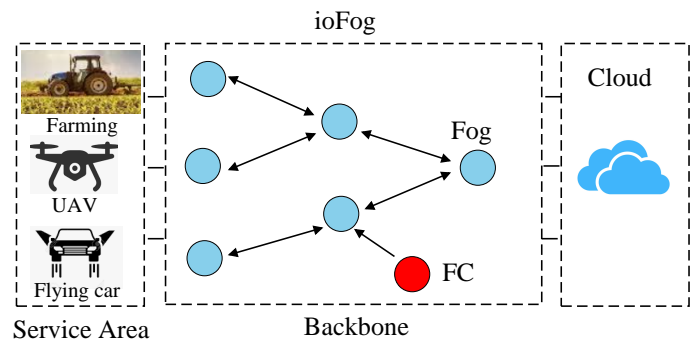

Fig. 1. Overview architecture of the ioFog

\section{A. Motivations}

The evolution of Software-Defined Networking (SDN) plays a significant role in the development of Next-Generation Networks (NGN) [4]. This increases flexibility by reducing complexity in networks. Taking the advantages, we can deploy $F C$ platforms over the same network solution for improved fog computing services. Similarly, the backbone device/router has computing capacity. Fog computing approaches in IoT bring several benefits in reducing latency, improved resiliency and availability, lower costs, and local data storage [5]. The outcomes are necessary for various critical IoT applications. For example, latency can be a matter of death for applications like healthcare and autonomous vehicles. These applications require the highest level of availability and resiliency to ensure the safety and continuity of services. Moreover, edge computation can save an organization's bandwidth by not sending all the IoT traffic towards the Internet cloud.

While provisioning resiliency and availability, offline connectivity is essential for critical application in the considered network scenarios. For example, in the case of a refinery gas leakage detection system, it must operate without Internet access. Vehicular networks, Unmanned Aerial Vehicles (UAVs), and rural networks use long-distance and multi-hop wireless links for Internet connections. Edge- and hierarchical fog-based architecture show suitable for improved decisionmaking [6]. However, these solutions hardly provide services, specially when the requirements are dynamic in nature. Different localized computing platforms, such a Dew computing, 
consider individual fog modules for an offline solution [7]. However, it fails when it comes to distributed monitoring and analysis. Ultra-low analytic and offline task management is a challenge. With the growing number of fog nodes, network and computation service management is an intricate issue.

\section{B. Contributions}

In this paper, we propose a new Fog computing architecture for offline IoT operations. The proposed architecture uses the prediction-based approach to improve the task processing operation in fog nodes and the cloud. Based on the user's task requirements patterns, a fog controller module in the network makes offline operation available. In summary, the key contributions of this paper are as follows:

- We propose a fog controller-based architecture for improved task processing among the computing modules in fogs and clouds.

- We propose a prediction-based computation monitoring and control mechanism for ioFog.

- An offline computation mechanism is proposed for the fog nodes to support availability and resilience services in IoT.

\section{RELATED WORK}

In the literature, significant research has been focused on enhancing task offloading and resource allocations in the fog computing paradigm. It has been observed the major concerns in task offloading are latency aware, energy-aware, resource-aware, deadline-aware, priority-aware, privacy-aware, and mobility-aware. There are three different offloading mechanisms possible, namely terminal-fog offloading, fog-fog offloading, and fog-cloud offloading. Zhang et al. [8] introduced one energy and delay minimized offloading scheme based on overall energy consumption in the network, historical energy consumption, and priority of the fog node. The task is split into two, one has to process locally at the terminal node, and the other has been processed at fog node. Misra et al. [9] considered an SDN-based multi-hop task offloading policy aiming to reduce delay and energy consumption of the network. Adhikari et al. [10] has addressed deadline and priority aware task offloading issue by considering the fog-cloud environment where the priority is being checked at IoT gateway and by following the deadline, latency is minimized on performing computation at suitable fog node. The selection of a suitable Fog node to perform task offloading and computation has a critical role in Fog network scenario. Game theory-based technique is adopted to tackle fog node selection problem [11], [12].

Resource allocation has a prime role in the computation at fog node. Task offloading at fog node considering resources makes the network more efficient [12][13]. Some literature proposes energy-efficient resource management schemes for coordination computation, such as offloading among fog computing devices. In [14], the authors addressed the computation offloading mechanism for fog Radio Access Network (RAN) along with a task offloading algorithm is proposed based on parallel communication and computation for radio access network claiming steady scalability and efficiency. [15] also considered a 5G fog radio access network to tackle offloading decision challenge and minimize energy and delay. Taking the energy minimization factor into consideration, the authors in [16] has come up with a significant probabilistic fogcloud computation model based on popularity distribution of cloud tasks. Delay optimization in task offloading makes the fog network more scalable and efficient, serving the primary purpose of latency reduction for fog computing. Extensive research has been performed to reduce the overall delay in performing offloading and computation [9], [17].

Dew computing is to enable ubiquitous, convenient readyto-go and plug-in facility for personal area network aiming to provide access and network activities offline [7]. Extensive research can be found on resource-sharing fog nodes in a fog network for offloading tasks and performing the computation in distributed scenery. Still, no work is done yet for considering a centralized fog controller to provide an offline facility as far our intense research yet.

Synthesis: Based on the above analysis, we infer the following:

- The existing solutions are deficient in reliability and resiliency for critical IoT applications running the largescale and wireless backbone network.

- The current fog-based task offloading solutions require a massive amount of control overhead and fail to provide low latency and real-time services.

- The unavailability of prediction-based service architecture, the current fog-based schemes are not sufficient for provisioning ultra-low latency analytic and decisions.

Eventually, there emerges a need for a fast and intelligent fog-network.

\section{IOFOG: THE PROPOSED ARCHITECTURE}

This section discusses the proposed fog network architecture. We discuss ioFog into two subsections - (i) System model for ioFog, and (ii) Markov chain model for task prediction.

\section{A. System Model}

We consider a smart centralized fog controller $(F C)$ enabled IoT network for monitoring and allocating service with minimum latency. As shown in Fig. 2, we consider a network consisting a set of fog nodes $F=\left\{F_{1}, F_{2}, F_{3}, \ldots, F_{n}\right\}$ to perform various computations at the fog-layer of the architecture and a set of terminal nodes $T=\left\{T_{1}, T_{2}, T_{3}, \ldots, T_{m}\right\}$ in the network for end-user operations. The fog computing modules are deployed over the capable computing device in the backbone network.

The terminal nodes are categorized as per their services, such as smart-home services, health services, and smartcity services. Our architecture's Fog controller is assumed to be rich in computation and energy, connected to all the fog nodes in wireless modes. Fog computer is a centrally connected fog node with superior resource availability for computation and not energy-constrained. The global view of 


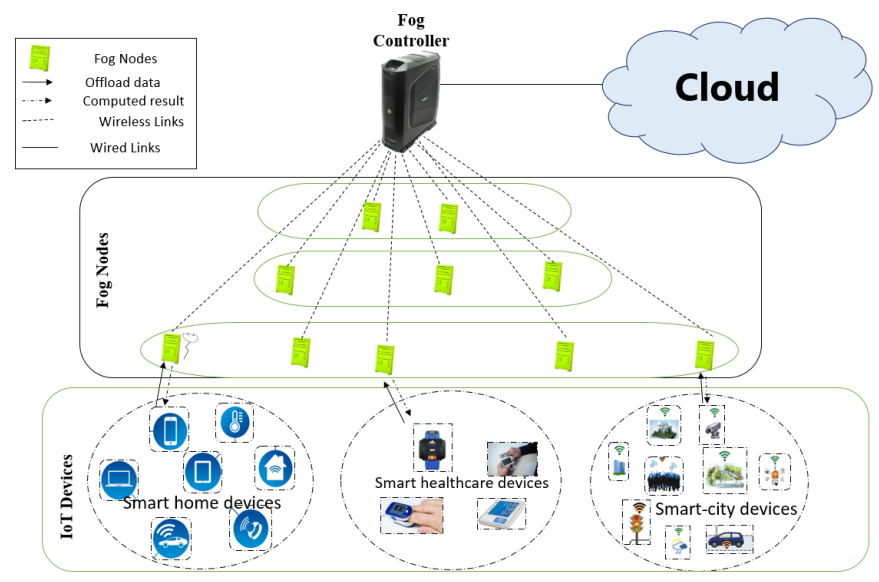

Fig. 2. Architecture of ioFog

the fog nodes is achieved through an SDN controller with a status update mechanism. FC collects network views from the SDN controller, which is installed side by side in the same system. Whenever there is a request from a terminal node, the nearest fog node termed as primary fog node $\left(P_{i}\right)$ checks its requirement. If $P_{i}$ can serve the request, it sends a copy of the available resource status to $F C$. Otherwise, $P_{i}$ sends a request to $F C$ for deciding which fog node to forward. The $F C$ takes this decision by taking care of optimal delay and energy consumption. Additionally, $F C$ uses the Markov-chain model for predicting the arrival of a task type to provide faster and offline services. We discuss the proposed Task allocation and Prediction model, and performance models delay, computation, and energy below.

1) Task Allocation Model: For task allocation, we need to inspect the various capabilities of the devices such as CPU, GPU, energy, and storage capacity. We consider the $F C$ has enriched in terms of computation serving prediction-based facility and also energy sustainability. FC has the global status of fog nodes concerning the availability of resources. Accordingly, it can decide the most suitable fog node for performing a task with its required amount of resources. Further, FC uses Markov chain-based prediction to find the type of tasks and takes allocation decisions as per their criticality measures.

Although we consider $F C$ as a reliable system, single-pointof-failure questions the resiliency in the proposed architecture. To tackle this issue, after requesting a task allocation, the Primary Fog node $\left(P_{i}\right)$ waits for an acknowledgment from the $F C$ asking for confirmation. If it fails to receive any response, it forwards the same to the Cloud. However, in such a case, immediate service provisioning is not possible in offline mode.

2) Delay Model: Delay is a crucial factor that needs to be addressed and optimized according to obtain efficient network performance. The total delay is the sum of communication delay, which is computed as $\mathcal{D}_{\mathcal{T}}$ and computation delay as $\mathcal{D}_{\mathcal{C}}$.

Communication Delay: In the proposed model, the communication is set up among terminal nodes, fog nodes, fog controller, and the cloud. Considering each terminal node has some tasks to compute, it is sent to the $P_{i}$ for computation. If $P_{i}$ fails then it communicates to the $F C$, which then chooses a best fog node for offloading. However, the task added at the fog node communicates the results to the terminal node on demand basis. We also assume that the terminal node serves the task to the fog node via a time-slotted mechanism. We split the communication process into data and control communication models. The path travelling from $T_{m}$ to $P_{i}$ to $P_{j}$ (secondary fog node) to cloud and reverse. This communication cost is measured by adding data and control communication cost. For control communication, cost incurs while sending information from fog node to FC and vice-versa. This cost also includes sending control information from the fog node to the terminal and cloud. Total communication delay is calculated from the addition of propagation delay $\left(\mathcal{D}_{P}^{c}\right)$ and transmission delay $\left(\mathcal{D}_{T}^{c}\right)$. The propagation delay is measured by counting the number of hops for transmitting the task request from terminal node to fog node and fog node to cloud as:

$$
\mathcal{D}_{P}^{c}=\sum_{T=1}^{i} P_{i}^{\{a\}}
$$

$$
\text { here, } \ddot{a}=a_{T i}^{P i}+a_{P i}^{P j}+a_{P i}^{C}+a_{C}^{T i}+a_{P j}^{T i}
$$

where, $\mathcal{D}_{P}^{c}$ is the propagation delay, $P_{j}$ is the secondary fog node, and $C$ is the cloud. $a_{T i}^{P i}, a_{P i}^{P j}, a_{P i}^{C}, a_{C}^{T i}, a_{P j}^{T i}$ finds the communication cost from $T_{i}$ to $P_{i}, P_{i}$ to $\left.P_{j}\right), P_{i}$ to $\mathrm{C}, \mathrm{C}$ to $T_{i}$ (optional) and $P_{j}$ to $T_{i}$ (optional), respectively. $P_{i}$ is the nearest fog node to $T_{i}$ and $P_{j}$ is the chosen fog node for offloading and computation. Transmission delay is a comprehensive transmission rate in the medium considering up and downtime for the tasks. Unlike other schemes in literature where the downtime is ignored because it brings the result, which is most likely a control signal, we consider the downloading time as well to predict future transmissions accurately. Transmission delay is calculated by adding delay of terminal node to $P_{i}$ as $\left(\mathcal{D}_{T}^{F}\right)$, from $P_{i}$ to $P_{j}\left(\mathcal{D}_{P_{i}}^{P}{ }_{j}\right)$ and fog node (primary or secondary or both) to cloud $\left(\mathcal{D}_{P i / P j}^{C}\right)$ along with downloading delay from $P_{j}$ to terminal node as $\left(\mathcal{D}_{P}^{T i}\right)$ and from cloud to terminal node as $\left(\mathcal{D}_{C}^{T i}\right)$.

The total transmission delay is calculated as:

$$
\begin{aligned}
& \mathcal{D}_{T}=\frac{\mathcal{D}_{T}^{F}+\mathcal{D}_{P_{i}{ }^{P} j}+\mathcal{D}_{P i / P j}^{C}}{\mathcal{B} \cdot E_{n}} \\
& E_{n}=\log _{2}\left(1+\frac{t p_{n} \cdot p l_{n} . s f_{n}}{I_{n}+B . n p}\right)
\end{aligned}
$$

where, $\mathcal{D}_{T}^{F}=\frac{A_{T i i}^{P} . L}{R_{i, P i}}, D_{P_{i}{ }^{P}}=\frac{B_{P_{i}{ }^{P} . L}}{R_{P_{i}, P_{j}}}$ and $D_{P i / P j}^{C}=\frac{C_{P_{i}}^{C} \cdot L}{R_{P_{i}, C}} . A_{T i}^{P}, B_{P_{i}{ }^{P}}, C_{P_{i}}^{C}$ are size of task data from $T_{i}$ to $P_{i}$, size of task from $P_{i}$ to $P_{j}$ and size of task from $P_{i}$ to $C$, respectively, $L$ is the link capacity, $R_{i, P_{i}}$, $R_{P_{i}, P_{j}}$ and $R_{P_{i}, C}$ denotes rate of transmission from fog node $n$ to $P_{i}$ and from $P_{i}$ to $P_{j}$ and from $P_{i}$ to $C$, respectively. $\mathcal{B}$ is the allocated bandwidth and $E_{n}$ is spectral efficiency 
of the wireless link from terminal node to fog node, i.e., obtained from Shanon's capacity. $t p_{n}, p l_{n}, P j_{n}, I_{n}$ and $n p$ are transmission power, path loss, shadowing factor, interface power, and noise power respectively.

Computation Model and Computation Delay: The task at a terminal node is calculated using (i) CPU cycle time and (ii) the size of data. Let $C_{i}$ be the CPU cycle for processing data and CPU frequency as $f_{i}$. Let $\mathcal{T}_{i}$ size of task is considered at the terminal node $i$ and $P_{i}$ be the total number of CPU cycles required for the computation. The value of $\mathcal{P}$ can be expressed as:

$$
\mathcal{P}_{i}=\mathcal{T}_{i} C_{i}
$$

Total task completion time $\left(T_{C}\right)$ can be obtained from the below mentioned expression:

$$
\mathcal{T}_{C}=\mathcal{T}_{T i, P i}^{t}+\mathcal{T}_{P i}^{P}{ }_{j}+\mathcal{T}_{T i}^{\text {comp }}+\mathcal{T}_{P j}^{T i}
$$

where $\mathcal{T}_{T i, P i}^{t}$ is the time taken to send $T_{i}, P_{i}$ task from terminal node to $P_{i}$ for $\mathcal{T}_{i}$ amount of data with $r_{i}$ rate of uplink, $T_{P_{i}{ }^{P}}$ from $P_{i}$ to $P_{j}, \mathcal{T}_{T i}^{\text {comp }}$ time required for computation at $P_{j}$, which is calculated as $\mathcal{T}_{T i}^{\text {comp }}=\frac{P_{i}}{f_{i}}$ ) and $\mathcal{T}_{P j}^{T i}$ for time cost of result from $P_{j}$ to terminal node. In literature this cost is neglected as it carries the size of data, which is more like a control signal. The computation delay $\left(\mathcal{D}_{C}\right)$ relies on CPU cycles and clock speed, i.e., processing density $(d p)$. Total computation delay is $\mathcal{D}_{C}=\mathcal{D}_{Q}+\mathcal{D}_{T P}$, here $\mathcal{D}_{Q}$ and $\mathcal{D}_{T P}$ are queuing delay and task processing delay.

$$
\begin{gathered}
\mathcal{D}_{Q}=\mathcal{D}_{Q i}=\sum_{T=1}^{n} Q_{P}^{n}{ }_{i}^{n, t s} \\
\mathcal{D}_{T P}=\frac{d p \cdot A_{T i}}{f_{i}}
\end{gathered}
$$

Here, $d p, f_{i}, Q_{P}^{n, t}$ are processing density, CPU clock speed of terminal node $i$ and number of task at $P_{i}$ at the time slot $s=\{1,2,3, \ldots, n\}$.

3) Energy Model: Although fog computing has a remarkable contribution towards the IoT networks paradigm, it has a weighty drawback due to its energy-constrained devices. Although technology has emerged exponentially, the technology regarding battery-powered devices still lacks sufficient energy without limiting the processor's clock speed. In fog applications, they desire to have low latency and high computational facilities that can not afford by the nodes themselves due to energy, computation, and storage constraints. The energy consumption in each state of the network service is calculated per cycle time proportional to the frequency. It is expressed as $\mathcal{E}=\left(F_{i}\right)^{2} P_{i}$. However, for each device, $\mathcal{E}$ value has been provided by the manufacturer. For overall energy consumption in the operations, we assume $\mathcal{E}_{n}$ brings all the energy consumption together in the network at each state, such as energy consumption during transmission $\mathcal{E}_{t}$, idle time $\mathcal{E}_{i}$, receiver $\mathcal{E}_{r}$ and at sleep mode $\mathcal{E}_{s l}$. Total energy consumption can be calculated as:

$$
\mathcal{E}_{\text {total }}=\sum_{T=1}^{n} \mathcal{E}_{t} \times P_{t}+\mathcal{E}_{i} \times P_{i}+\mathcal{E}_{r} \times P_{r}+\mathcal{E}_{s l} \times P_{s l}
$$

It is to be noted that $P_{t}, P_{i}, P_{r}$ and $P_{s l}$ are the power needed for $\mathcal{E}_{t}, \mathcal{E}_{i}, \mathcal{E}_{r}$ and $\mathcal{E}_{s l}$ are fixed for each devices.

\section{B. Markov Prediction-based Offline IoT}

The ioFog architecture performs operations - (1) any task generated on the terminal node at a specific time slot is sent to the $P_{i}$, (2) $P_{i}$ informs about the incoming task to $F C$, (3) $F C$ chooses $P_{j}$ using it's prediction-based trained model and informs $P_{i}$, (4) $P_{i}$ forwards the task to the $P_{j}$ (5) after processing the data at $P_{j}$, it sends a result to the terminal node if required and a copy to the cloud. Till this point, no offline computation happens. Based on the repeated transaction on a particular fog node using a prediction algorithm at $F C$, it keeps complete information of fog nodes. The $F C$ predicts the next probable transaction service of types and sends the information of prediction to the $P_{i}$. Receiving any task at $P_{i}$ as per the prediction, it forwards the task, and the $P_{j}$ performs its computation and sends the result to the terminal node in offline mode. As soon as the Internet gets reconnected, everything gets synchronized accordingly. Fig. 3 shows the steps for the proposed prediction-based task allocation scheme.

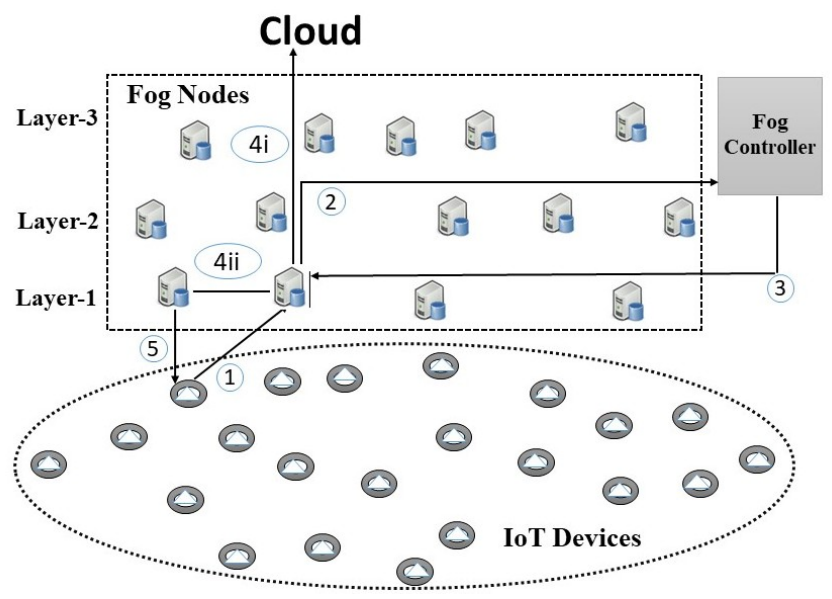

Fig. 3. Working of ioFog

We use Markov predictor with order- $\mathrm{x}, \mathrm{O}(\mathrm{x})$ to predict the future task type at the $P_{i}$, which exaggerates our prime objective to maximize and reliable service while the network goes offline along with optimizing the delay and energy consumption. The model consists of two tuples $\left(H_{i}, p\right)$. Here $H_{i}$ is the history of the type of tasks received at that $P_{i}$ and the probability of a task $p$.

- $H_{i}$ : This holds three tuples $\langle M, A\rangle$ where $M$ implies the set of task types, denoted by $M=\left\{m_{1}, m_{2}, m_{3}, \ldots, m_{i}\right\}$. $A$ is the time of task arrival at the $P_{i}$, denoted by $A=\left\{a_{1}, a_{2}, a 3, \ldots, a_{i}\right\}$. 
- $p$ : It is further divided in to $p_{i}$ and $p_{j}$ which defines the probability of type of the task and arrival time, respectively.

The tuples used above are to get the prediction by the $F C$ at one specific $P_{i}$. Similarly, for all other fog nodes in the network, we perform these operations too.

Assumption As the $F C$ is connected to all the fog nodes. It collects all the information, such as types of service receiving and time of a received task.

Definition 1 (Task). A sensor collects its parameters from the surroundings, such as a healthcare IoT device that collects reading of oxygen level, temperature, and heartbeat rate of a human body. Here we have three categories of tasks available; one is computation on oxygen level, rate of heartbeat, and computation on temperature. The set of tasks $M$ are presented as above.

Definition (Arrival Time at $P_{i}$ ) The task generated at the terminal node is sent to the $P_{i}$. Here the time of arrival of each task is treasured to have a record.

Corollary. Let us consider the Markov predictor with order $x=3$ is a finite state predictor with $2^{x}$ possible types of tasks, while considering $i=0$ in $M(i-x+1, i)=M_{i-2}, M_{i-1}, M_{i}$, predictors performance is not degraded.

We calculate the probability of task type for a specific period which arrives at the $P_{i}$. The time period is considered as $t_{x}$ and type of task at present is $m$. The previous record of the tasks is pulled from $M=m_{1}, m_{2}, m_{3}, \ldots, m_{i}$, and from $M$, the order- $x$ task type, we calculate $l_{c}=M(i-$ $x+1, i)=M_{i-2}, M_{i-1}, M_{i}$. Using the $l_{c}$ the task predictor finds similarity with calculated $l_{c}$. When the similar task is found, period of time is calculated as

$$
t p_{m}=t_{p} \mid t_{p}=t_{i+1}-t_{i},
$$

Here $t p_{m}$ is the time period for $m$. Then we calculate the conditional probability $P_{m}\left(t_{p} \leq t_{i}<t_{i}+t_{x}\right)$, that the task type is changed in $t_{x}$ time after time $t_{i}$. Similarly for $l_{c}$ and $t_{i}$, we find the change of task type at each $P_{i}$ is shown below:

$$
P\left(m \mid l_{c}, t_{i}\right)=P(m) P_{m}\left(t_{i} \leq t_{p}<t_{i}+t_{x} \mid l_{c}, t_{i}\right)
$$

here $P_{m}$ is the probability of immediate future task, we derive as

$$
P\left(m_{t+1}=m \mid H_{i}\right) \approx P\left(m_{t+1}=m \mid H_{i}\right)=\frac{A\left(l_{c m}, H_{i}\right)}{A\left(l_{c m}, H_{i}\right)}
$$

$A\left(l_{c m}, H_{i}\right)$ is the calculation of how many times the $l_{c}$ has come in the record of $H_{i}$. The future task is predicted using Markov model for different task $M$, lets consider the next task is $t_{i+1}$ then the next task type is as Equation (11) below:

$$
m_{t+1}=\operatorname{argmax}\left(P\left(m_{t+1}=s\right)\right)
$$

From Algorithm 1, we predict the next task type in a $P_{i}$. However, this algorithm works concurrently for all the available fog nodes in the network.

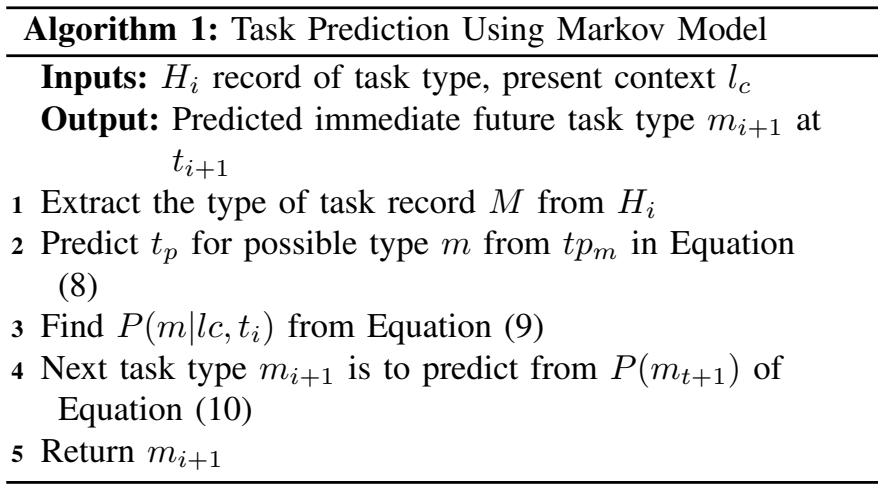

\section{Performance Evaluation}

We use iFogSim [18] simulator for analysis of the fog network (as shown in Fig. 3). We create a set of dynamic service requests to the network (i.e., to the fog nodes), and backbone communication links are considered WiFi-based long-distance point-to-point links [6]. We compare ioFog with the existing benchmarks - Fog and Cloud architecture.

\section{A. Prediction Accuracy}

The prediction of the type of next task to reach the $P_{i}$ at a specific period based on the history of its previous occurrences is made. Fig. 4 shows the prediction accuracy for three different tasks - (i) temperature, (ii) heartbeat, and (3) oxygen level of the human body, which is predicted for six times using Markov-prediction. Targeting a predicted task at a particular time and to provide service to the network offline is well performed in this evaluation, which provides accuracy up to $68 \%$.

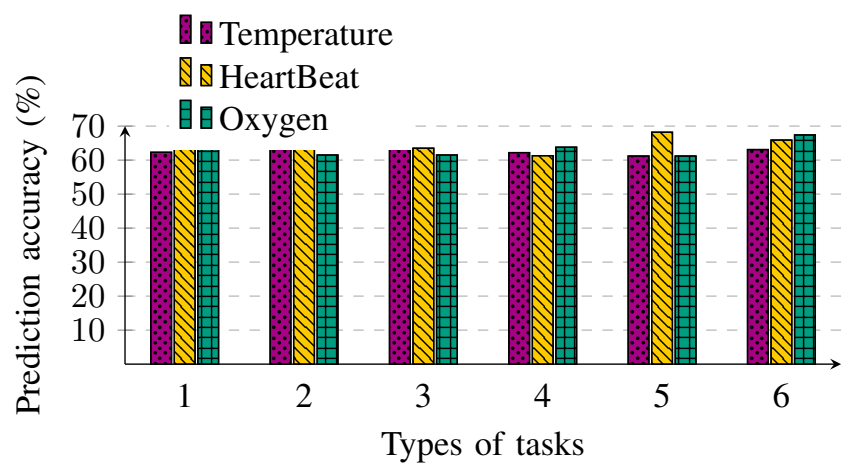

Fig. 4. Prediction Accuracy

\section{B. Service Time Analysis}

Due to localized decision and offline solution service mechanism, our solution significantly reduces latency, considering the proposed multi-level fog network. For $\gamma$ number of levels, decision time, $\mathcal{D}_{t} \propto L$. In the cloud case, the latency increases, and the maximum portion of it comes from the Internet. Fig. 5 shows the average service time in the proposed and cloud architecture. On the other hand, the existing fog and 
cloud architecture do not support prediction-based service provisioning for low latency operations.

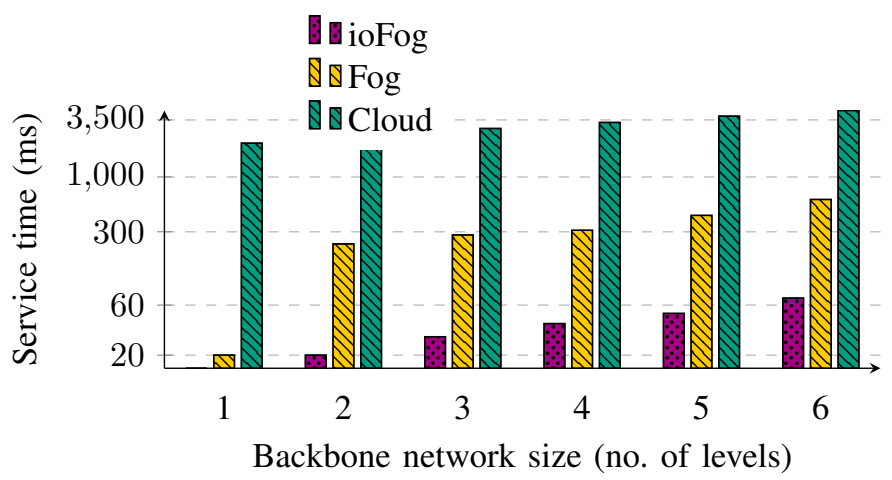

Fig. 5. Average service time for multiple level-based fog network

\section{Reliability Analysis}

We analyze the reliability of the proposed solution with the dynamic service request to the fog nodes. As shown in Fig. 6, the service delivery ratio in the proposed scheme offers almost 99\%. As the existing fog and cloud solution fails to predict the network's future task requirements, these fail to provide services when the network goes offline.

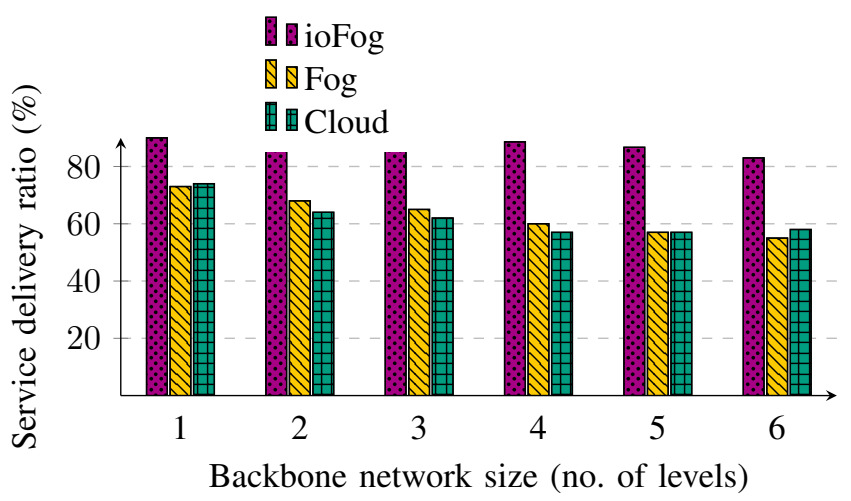

Fig. 6. Average service delivery ratio for multiple level-based fog network

\section{CONCLUSION}

This paper presented a fog-computing architecture ioFog, to overcome the drawbacks of unreliable connections at the backbone network. The drawbacks are mainly related to unreliable, long-distance, and wireless backbone, and dynamic service requirements. First, we proposed a controller-based fog network architecture to remedy these limitations, which maximizes the service provisioning in offline mode. The proposed architecture used a prediction-based approach to predict a future task and pushed it to a target-specific fog node dynamically. Once a task is made available on a dynamic and prediction model, the user can get the requested service without internal connectivity. Our solution shows better service delivery and time results as compared to the existing state-ofthe-art.
As future works, we will work to increase the prediction accuracy of an incoming task and optimize resource utilization using machine learning approaches. In addition to this, we plan to deploy the proposed scheme over a real IoT testbed setup.

\section{REFERENCES}

[1] M. Mukherjee, L. Shu, and D. Wang, "Survey of fog computing: Fundamental, network applications, and research challenges," IEEE Communications Surveys \& Tutorials, vol. 20, no. 3, pp. 1826-1857, 2018.

[2] Y. Xiong, Y. Sun, L. Xing, and Y. Huang, "Extend cloud to edge with KubeEdge," in 2018 IEEE/ACM Symposium on Edge Computing (SEC), pp. 373-377, IEEE, 2018.

[3] O. Ali and M. K. Ishak, "Bringing intelligence to IoT Edge: Machine Learning based Smart City Image Classification using Microsoft Azure IoT and Custom Vision," in Journal of Physics: Conference Series, vol. 1529, p. 042076, IOP Publishing, 2020.

[4] A. A. Barakabitze, A. Ahmad, R. Mijumbi, and A. Hines, "5G network slicing using SDN and NFV: A survey of taxonomy, architectures and future challenges," Computer Networks, vol. 167, p. 106984, 2020.

[5] I. Martinez, A. S. Hafid, and A. Jarray, "Design, Resource Management and Evaluation of Fog Computing Systems: A Survey," IEEE Internet of Things Journal, 2020. doi: 10.1109/JIOT.2020.3022699.

[6] N. Ahmed, D. De, and I. Hussain, "Internet of Things (IoT) for smart precision agriculture and farming in rural areas," IEEE Internet of Things Journal, vol. 5, no. 6, pp. 4890-4899, 2018.

[7] P. P. Ray, "An introduction to Dew computing: definition, concept and implications," IEEE Access, vol. 6, pp. 723-737, 2017.

[8] G. Zhang, F. Shen, Z. Liu, Y. Yang, K. Wang, and M.-T. Zhou, "FEMTO: Fair and Energy-minimized task offloading for fog-enabled IoT networks," IEEE Internet of Things Journal, vol. 6, no. 3, pp. 43884400, 2018.

[9] S. Misra and N. Saha, "Detour: Dynamic task offloading in softwaredefined fog for iot applications," IEEE Journal on Selected Areas in Communications, vol. 37, no. 5, pp. 1159-1166, 2019.

[10] M. Adhikari, M. Mukherjee, and S. N. Srirama, "Dpto: A deadline and priority-aware task offloading in fog computing framework leveraging multilevel feedback queueing," IEEE Internet of Things Journal, vol. 7, no. 7, pp. 5773-5782, 2020.

[11] Z. Liu, Y. Yang, K. Wang, Z. Shao, and J. Zhang, "Post: parallel offloading of splittable tasks in heterogeneous fog networks," IEEE Internet of Things Journal, vol. 7, no. 4, pp. 3170-3183, 2020.

[12] J. Baek and G. Kaddoum, "Heterogeneous task offloading and resource allocations via deep recurrent reinforcement learning in partial observable multi-fog networks," IEEE Internet of Things Journal, 2020. doi: 10.1109/JIOT.2020.3009540.

[13] Y.-L. Jiang, Y.-S. Chen, S.-W. Yang, and C.-H. Wu, "Energy-efficient task offloading for time-sensitive applications in fog computing," IEEE Systems Journal, vol. 13, no. 3, pp. 2930-2941, 2018.

[14] K. Guo, M. Sheng, T. Q. Quek, and Z. Qiu, "Task offloading and scheduling in fog ran: A parallel communication and computation perspective," IEEE Wireless Communications Letters, vol. 9, no. 2, pp. 215-218, 2019.

[15] Z. Cheng, Y. Tang, and H. Wu, "Joint task offloading and flexible functional split in 5g radio access network," in 2019 International Conference on Information Networking (ICOIN), pp. 114-119, IEEE, 2019.

[16] J. Kim, T. Ha, W. Yoo, and J.-M. Chung, "Task popularity-based energy minimized computation offloading for fog computing wireless networks," IEEE Wireless Communications Letters, vol. 8, no. 4, pp. 12001203, 2019.

[17] M. K. Hussein and M. H. Mousa, "Efficient task offloading for iotbased applications in fog computing using ant colony optimization," IEEE Access, vol. 8, pp. 37191-37201, 2020.

[18] H. Gupta, A. Vahid Dastjerdi, S. K. Ghosh, and R. Buyya, "iFogSim: A toolkit for modeling and simulation of resource management techniques in the Internet of Things, Edge and Fog computing environments," Software: Practice and Experience, vol. 47, no. 9, pp. 1275-1296, 2017. 